\title{
High Glucose-Induced Podocyte Injury Involves Activation of Mammalian Target of Rapamycin (mTOR)-Induced Endoplasmic Reticulum (ER) Stress
}

\author{
Jie Lei ${ }^{\mathrm{a}} \quad$ Lei Zhao $^{\mathrm{a}} \quad$ Yujing Zhang ${ }^{\mathrm{b}} \quad$ Yanfeng Wu $\mathrm{W}^{\mathrm{c}}$ Yanbo Liu ${ }^{\mathrm{a}}$ \\ aDepartment of Pediatrics, the First Hospital of Jilin University, Changchun, Jilin, ${ }^{b}$ Department of \\ Pediatrics, the Second Affiliate Hospital of Harbin Medical University, Harbin, Heilongjiang, 'Department \\ of Pneumology, the Second Hospital of Jilin University, Changchun, Jilin, PR China
}

\author{
Key Words \\ Podocyte $\bullet$ High glucose $\cdot$ Apoptosis $・$ ER stress $\bullet$ mTOR
}

\begin{abstract}
Background/Aims: The mechanisms by which high glucose (HG) results in podocyte damage remains unclear. We investigated the potential role of endoplasmic reticulum (ER) stress and mTOR signaling in HG injured podocyte. Methods: In cultured mouse podocytes, cellular apoptosis was assessed using FITC-Annexin $V$ and propidium iodide staining followed by flow cytometry analysis. Apoptosis-related proteins as well as the ER stress and the mTOR signals were evaluated using immunoblot assay. Results: Compared to normal glucose (NG) and osmotic mannitol (MN) control, the percentage of apoptotic cells was increased significantly in HG-treated podocytes. The levels of CHOP, Grp78, phospho-PERKThr982, and caspase-12 were increased significantly following HG treatment. The downstream effects of ER stress were obtained in HG-treated podocytes, showing upregulation of Bax, Bak and cytochrome $\mathrm{c}$, and downregulation of Bcl-2. HG-induced increase of cytochrome $\mathrm{C}$, Bax and active caspase-3 was prevented by both ER inhibitor sodium 4-phenylbultyrate (PBA) and CHOP siRNA (siCHOP). PBA and CHOP knockdown remarkably decreased HG-induced apoptosis. In addition, the levels of phospho-mTORSer2448 and phospho- p70S6kThr389 as well as phospho-AMPK $\alpha$ (a sensor of energy consumption) were increased significantly in HG-treated cells. Moreover, the Erk inhibitor U0126 prevented HG-induced mTOR activation. Increased phospho-AMPK $\alpha, \mathrm{CHOP}$ and Grp78 as well as cellular apoptosis were prevented by mTOR inhibitor rapamycin in HG-treated podocytes. Conclusion: Our data demonstrate that the activated mTOR by Erk1/2 results in energy consumption, which in turn leads to ER stress signaling and thus induces apoptosis in HG-treated podocytes.
\end{abstract}




\section{Cellular Physiology Cell Physiol Biochem 2018;45:2431-2443

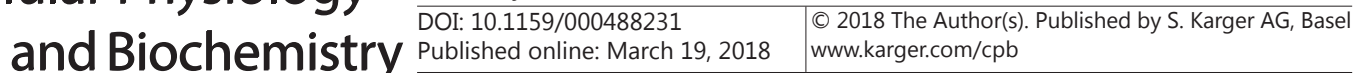

Lei et al.: mTOR Activation and ER Stress in HG Podocyte Injury

\section{Introduction}

Diabetes, a highly prevalent disease, can cause a progressive kidney glomeruli disease, termed as diabetic nephropathy (DN) or diabetic kidney disease (DKD). DN is the leading cause of end- stage renal failure that finally needs transplantation or dialysis [1]. Proteinuria is the major manifestation of DKD. Research demonstrates that glomerular podocyte injury is a key event leading to proteinuric kidney disease including DN [1,2]. Podocyte, a highly specialized epithelial cell, has a complicated cellular architecture with a large cell body seating on glomerular basement membrane by extending tons of foot processes (FPs) [3]. Morphologically, podocyte damage is mainly presented with FPs fusion/effacement, hypertrophy, detachment, loss and/or death [3]. As terminally differentiated epithelial cells, podocyte loss is generally considered as an irreversible event that results in a decline in glomerular filtration function [1-3]. Although it was reported that hyperglycemia or longterm blood high glucose (HG) can cause podocyte injury via a variety of cellular events such as oxidative stress, reactive oxygen species (ROS), and inflammatory cytokine production during the early pathogenic process of DN [4], the underlying molecular mechanisms by which HG results in podocyte injury have not yet been clarified.

Podocyte loss may occur via apoptotic cell death pathway in several DN animal models [5-7]. In cultured podocyte cell line, it was reported that podocytes underwent apoptosis under HG conditions [5, 6, 8-10]. Induction of p38MAPK caused by the release of mitochondrial and plasma membrane ROS as well as increased NADPH oxidase (NOX) plays a crucial role in HG-induced podocyte apoptosis [11]. In DN, the TGF $\beta$ level is upregulated, which induces podocyte apoptosis by activating caspase 3 through p38MAPK and Smad 7 as well as Notch and Wnt/ $\beta$ - catenin signaling $[12,13]$. Recently, it was found that decline of autophagy flux increases the ubiquitin-proteasome-dependent degradation pathway that is responsible for cell death in DN podocytes [14]. In addition, inflammatory pathways may be involved in podocyte loss in DN [15]. Notably, endoplasmic reticulum (ER) stress is an important cellular response that is required for process of unfolded and/or misfolded proteins in cell [16]. Over-activated or prolonged ER stress may initiate cellular apoptosis through two principal unfolded protein response (UPR) receptors inositol-requiring enzyme (IRE-1) and protein kinase RNA-like ER kinase (PERK) $[16,17]$. It was reported that oxidative stress and ER stress pathways participate in Aldo-induced podocyte injury [18]. Notably, research shows that a cross talk exists between ER stress and the mammalian target of rapamycin (mTOR) signaling, another important signaling pathway that regulates cell survival $[19,20]$. In podocytes, mTOR complex 2/Akt/NFKB-mediated activation of selective calcium ion channel protein TRPC6 involves in adriamycin-induced apoptosis [21].

In this study, we investigated the molecular mechanisms of HG-induced podocyte apoptosis by focusing on ER stress and mTOR signals. Increased ER stress and mTOR activity were detected in HG-treated podocytes. We also demonstrate that enhanced mTOR signaling by Erk1/2 contributes to ER stress activation through increasing energy consumption, which is related to HG-induced podocyte apoptosis.

\section{Materials and Methods}

\section{Primary antibodies}

The following primary antibodies were used: mouse anti-caspase-3, rabbit anti-cleaved caspase-3, and rabbit anti-caspase-12 (Abcam); rabbit anti-CHOP and mouse anti-Grp78 antibodies (Cell Signaling Technology); rabbit anti-mTOR, rabbit anti-phospho-mTORSer2448, and rabbit anti- phosphomTORSer2481 (Abcam); mouse anti-PERK and rabbit anti-phospho-PERKThr982 (Santa Cruz), rabbit anti-Bax, mouse anti-Bak, rabbit anti-Bcl-2, and rabbit anti-cytochrome c (Cell Signaling Technology); rabbit anti-P70S6K and rabbit anti-phospho-p70S6KThr389 (Abcam); rabbit anti-phospho-AMPK $\alpha$ Thr172, rabbit anti-AMPKo, rabbit anti-phospho-Erk1/2Thr202/Tyr204 and mouse anti-Erk1/2 (Cell Signaling Technology); and mouse anti- $\beta$-actin antibody (Sigma- Aldrich-Aldrich). 


\section{Cellular Physiology Cell Physiol Biochem 2018;45:2431-2443 and Biochemistry Published \begin{tabular}{l|l} 
DOI: 10.1159/000488231 & $\begin{array}{l}\text { C) } 2018 \text { The Author(s). Published by S. Karger AG, Basel } \\
\text { www.karger.com/cpb }\end{array}$ \\
\hline
\end{tabular}}

Lei et al.: mTOR Activation and ER Stress in HG Podocyte Injury

Cell culture and treatment

As previously described [22], immobilized mouse podocytes (a kind gift from Prof. Peter Mundel) were cultured at 33oC in RPMI1640 media containing fetal bovine serum (10\%, Invitrogen) and recombinant mouse interferon $-\gamma(10 \mathrm{U} / \mathrm{ml}$, Sigma-Aldrich-Aldrich). Following overnight serum starvation, the medium was replaced with RPMI1640 containing normal glucose (NG: $5.6 \mathrm{mM}$ ) or high glucose (HG: $30 \mathrm{mM}$ ). In addition, the cells that were treated with $24.4 \mathrm{mM}$ mannitol plus normal glucose were used as an osmotic control (MN). At the indicated time points, cells were collected for apoptosis analysis and immunoblotassay.

\section{CHOP knockdown}

To knockdown the expression of CHOP, we constructed three distinct plasmids psiRNAhH1GFPzeo (Invitrogen) that express a short interference RNA (siRNA) targeting to mouse CHOP gene (NM007837), respectively. The target sequence is siCHOP: 5'- gattccagtcagagttctatg-3', siCHOP1: 5'-aaggaagaactaggaaacgga-3', and siCHOP2: 5'-acgagcggaaagtggcacagc-3'. Podocytes were transfected with siCHOP plasmids using Lipofectamine 2000 (Invitrogen). CHOP knockdown efficiency was assessed using immunoblot assay $48 \mathrm{~h}$ after transfection. As compared to control plasmid (siControl), we obtained 77\%, $62 \%$ and $58 \%$ decline of CHOP protein level with siCHOP, siCHOP1 and siCHOP2, respectively. Thus, the plasmid expressing siCHOP was used in this study.

\section{Apoptotic detection}

As previously described [23], cells were stained with FITC-annexin V and propidium iodide (Sigma-Aldrich). The cells positively labeled with both FITC-anexin V and propidium iodide were considered as apoptotic cells that can be detected using flow cytometry (FACS). The percentage of apoptotic cells were calculated and compared.

\section{Immunoblot assay}

Total cellular protein was extracted using RIPA buffer containing freshly prepared protease and phosphatase inhibitor (Roche), and immunoblot assay was performed to evaluate the abundance of interested proteins [23]. Briefly, $75 \mu \mathrm{g}$ proteins each sample plus Laemmli loading buffer (final $1 \mathrm{x}$ with $0.1 \mathrm{M} \mathrm{DTT}$ ) were boiled for $5 \mathrm{~min}$. After high-speed centrifugation, the supernatant was collected and loaded on 4 - 15\% gradient SDS-PAGE. Protein was then electronically transferred to nitrocellulose membrane (Thermo Scientific). To reduce non- specific binding, membranes were incubated for $1 \mathrm{~h}$ in $5 \%$ BSA prepared with Tris-buffered saline containing 0.05\% Tween-20 (TTBS). Primary antibodies were applied to membrane and shaken for overnight in cold room. After 3 washes with TTBS, the membranes were incubated with HRP-conjugated goat anti-rabbit or mouse IgG. Finally, the blots were developed with an enhance ECL system (Pierce). The housekeeping gene encoded protein $\beta$-actin was used as the loading control. The specific band was scanned and quantified with Image J 1.4. The protein level relative to $\beta$-actin or total proteins was calculated, and the fold change over control was presented and compared.

\section{Statistical analysis}

Data are presented as the mean \pm stand deviation (SD). One-way ANOVA was used to compare the difference among different groups (Prism 4.0, GraphPad). The $p$ value equal to or less than 0.05 was regarded as significant difference.

\section{Results}

Over-activation of ER stress occurs in $H G$-induced podocyte injury

It has been shown that high glucose can induce podocyte injury [8-11, 24-26]. Accordingly, in comparison with normal glucose (NG) and osmotic mannitol (MN) control, we found that the percentage of apoptotic cells significantly increased at 12 (HG $5.93 \pm 0.603$ vs NG $2.17 \pm$ 1.00 and MN $2.47 \pm 0.901, p<0.05$ ), 24 (HG $11.17 \pm 1.061$ vs NG $2.10 \pm 0.562$ and MN $2.43 \pm 0.742, p<0.001)$ and $48 \mathrm{~h}(\mathrm{HG} 16.27 \pm 0.874$ vs NG $2.63 \pm 0.351$ and MN $2.97 \pm$ $0.351, p<0.001$ ) in high glucose (HG)-treated podocytes (Fig. 1A). To further confirm the effect of HG on podocyte apoptosis, we assessed the active caspase- 3 level by measuring the 
abundance of cleaved caspase- 3 , a general apoptosis marker [27]. Immunoblot assay detected a time-dependent increase of cleaved caspase-3 in HG-treated podocytes as compared with NG and MN controls (Fig. 1B). Although other groups also reported HG-induced podocyte apoptosis [8-11, 24], the underlying mechanisms are still elusive.

The endoplasmic reticulum (ER) stress, also termed as unfolded protein response (UPR), is a cellular response related to process of unfolded and/or misfolded proteins along with the increased production of molecular chaperones Bip/ Grp78 and CHOP [16, 17]. Over-activated or prolonged ER stress can initiate apoptosis through two principal UPR receptors IRE-1 and PERK $[16,17]$. In HG-treated podocytes, we found that the level of CHOP, Grp78 and the phosphorylated PERK at Thr389 was highly enhanced compared to NG- and MN-treated cells (Fig. 2). ER stress also activates caspase-12 through induction of m-calpain activation [28, 29], thus activating caspase- 9 followed by activation of caspase-3 [28-30]. In our study, increased caspase- 12 was also detected in HG-treated podocytes (Fig. 2).

The downstream signals of ER stress were further investigated. CHOP induces downregulation of the anti-apoptotic protein Bcl-2, causing mitochondrial damage, cytochrome c release and caspase-3 activation [31, 32]. Bax and Bak, the two pro-apoptotic proteins, are associated with the ER membrane. Under ER stress conditions, Bak and Bax trigger activation of the apoptotic caspase-3 pathway through release of $\mathrm{Ca} 2+$ to the cytosol [33-35]. In comparison with NG and MN controls, immunoblot assay showed that HG significantly increased the level of Bax, Bak and cytochromes c (Fig. 3). In consistent, anti-apoptotic protein Bcl2 level decreased remarkably in HG-treated cells (Fig. 3). Therefore, these findings

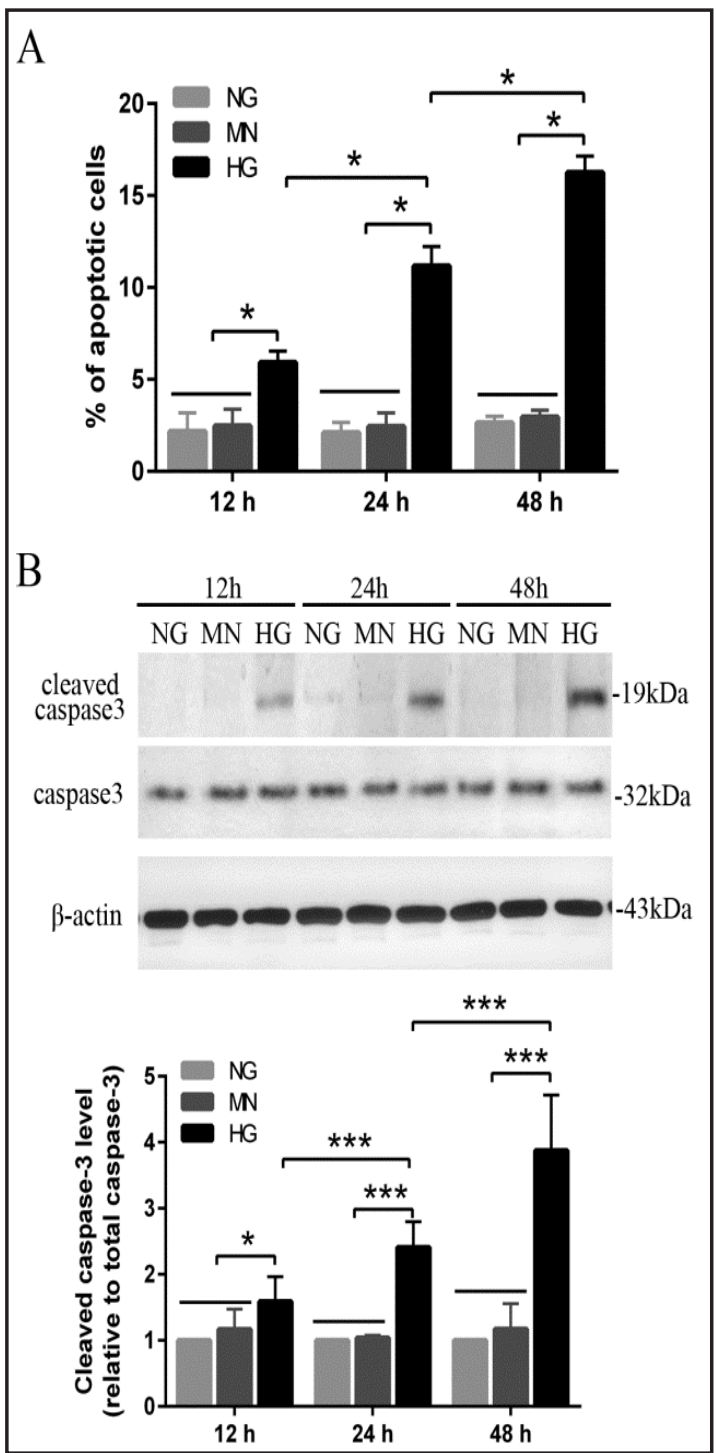

Fig. 1. HG treatment leads to apoptosis in cultured podocytes. Podocytes were treated with normal glucose (NG: $5.6 \mathrm{mM}$ ), mannitol and normal glucose (MN:24.4 mM), or high glucose (HG: $30 \mathrm{mM}$ ) for 12 , 24 , and 48 h, respectively. A. Cells were collected for apoptosis analysis. B. Total cellular protein was extracted and immunoblot assay was performed for evaluation of the cleaved caspase-3 level. Three independent experiments were performed. *: $\mathrm{p}<0.05$; $^{* * *}: \mathrm{p}<0.001$. suggest that ER stress is over-activated, which triggers its downstream signaling that may be involved in HG-induced podocyte apoptosis.

Over-activation of ER stress is responsible for HG-induced podocyte injury

To further elucidate the role of ER stress in HG-induced podocyte injury, the specific ER stress inhibitor sodium 4-phenylbultyrate (PBA) was applied as previously described [7]. Immunoblot assay showed that increased Grp78 and CHOP by HG was successfully 


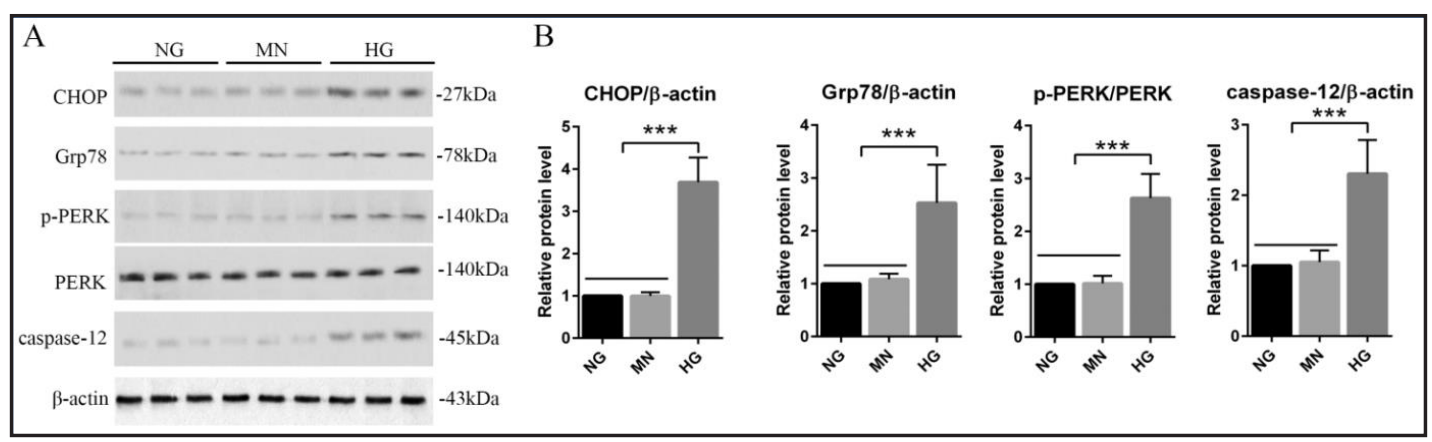

Fig. 2. Induction of ER stress occurs in HG-treated podocytes. Podocytes were treated for $24 \mathrm{~h}$ with 30 $\mathrm{mM}$ high glucose (HG). Normal glucose (NG: $5.6 \mathrm{mM}$ ) with or without mannitol (MN: $24.4 \mathrm{mM}$ ) treatment was used as control. A. Total cellular protein was extracted and immunoblot assay were performed for expression of endoplasmic reticulum (ER) stress proteins including CHOP, Grp78, phospho-PERK Thr982, and caspase-12. B. The specific band was scanned and quantitative analysis was performed with Image J. Experiments were performed in triplicate. ${ }^{* * *}$ : $p<0.001$.

prevented by PBA in a dosedependent manner (Fig. 4A). In HGtreated podocytes, the application of PBA prevented reduction of Bcl-2 and blocked increase of cytochrome c (Fig. 4B). This result demonstrates that HG-induced over-activation of ER stress and its downstream signaling was effectively inhibited by PBA. The effect of ER stress inhibition on podocyte injury was also studied in HG-treated cells, showing that HG-induced apoptosis $(13.07 \pm 1.05)$ was significantly $(p$ $<0.001)$ prevented by PBA $(10 \mathrm{mM}$ :

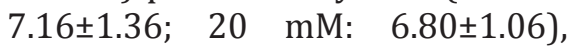
particularly at high concentration of 10 and $20 \mathrm{mM}$ (Fig. 4C).

In addition, we also applied siRNA that specifically targets to mouse CHOP to disrupt ER stress pathway. At protein level, HGinduced increase of CHOP was not detected in podocytes expressing siCHOP, but in cells expressing control siRNA (Fig. 4D). Notably, increased Bax and the cleaved caspase-3 were significantly prevented in HG-treated podocytes expressing siCHOP (Fig. 4E). Moreover, HG-induced apoptosis $(14.33 \pm 0.77)$ was remarkably $(p<$ $0.01)$ decreased by siCHOP $(7.33 \pm$

$0.84)$, not by siControl (14.03 \pm 1.08$)$ (Fig. 4F). Therefore, our data show that inhibition of ER stress by either PBA or siCHOP decreased HG-induced podocyte apoptosis, suggesting that over-activation of ER stress plays a critical role in HG-induced podocyte injury.

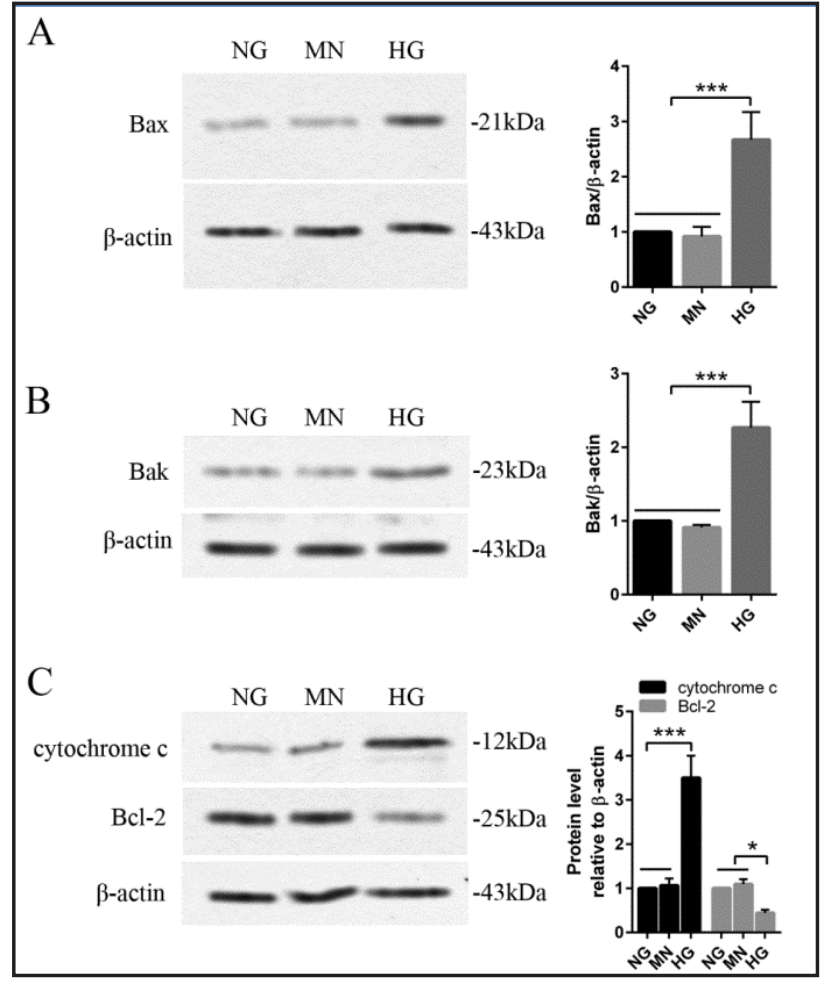

Fig. 3. Increased downstream signaling of ER stress is detected in HG-treated podocytes. Podocytes were treated for 24 h with high glucose (HG: $30 \mathrm{mM}$ ), and normal glucose (NG: $5.6 \mathrm{mM}$ ) with or without mannitol (MN: $24.4 \mathrm{mM}$ ), respectively. Total cellular protein was extracted and immunoblot assay was performed for expression of Bax (A), Bak (B), cytochrome $\mathrm{c}$ and Bcl-2 (C). Experiments were performed in triplicate. *: $\mathrm{p}<0.05$; ${ }^{* * *}$ : $\mathrm{p}<0.001$. 


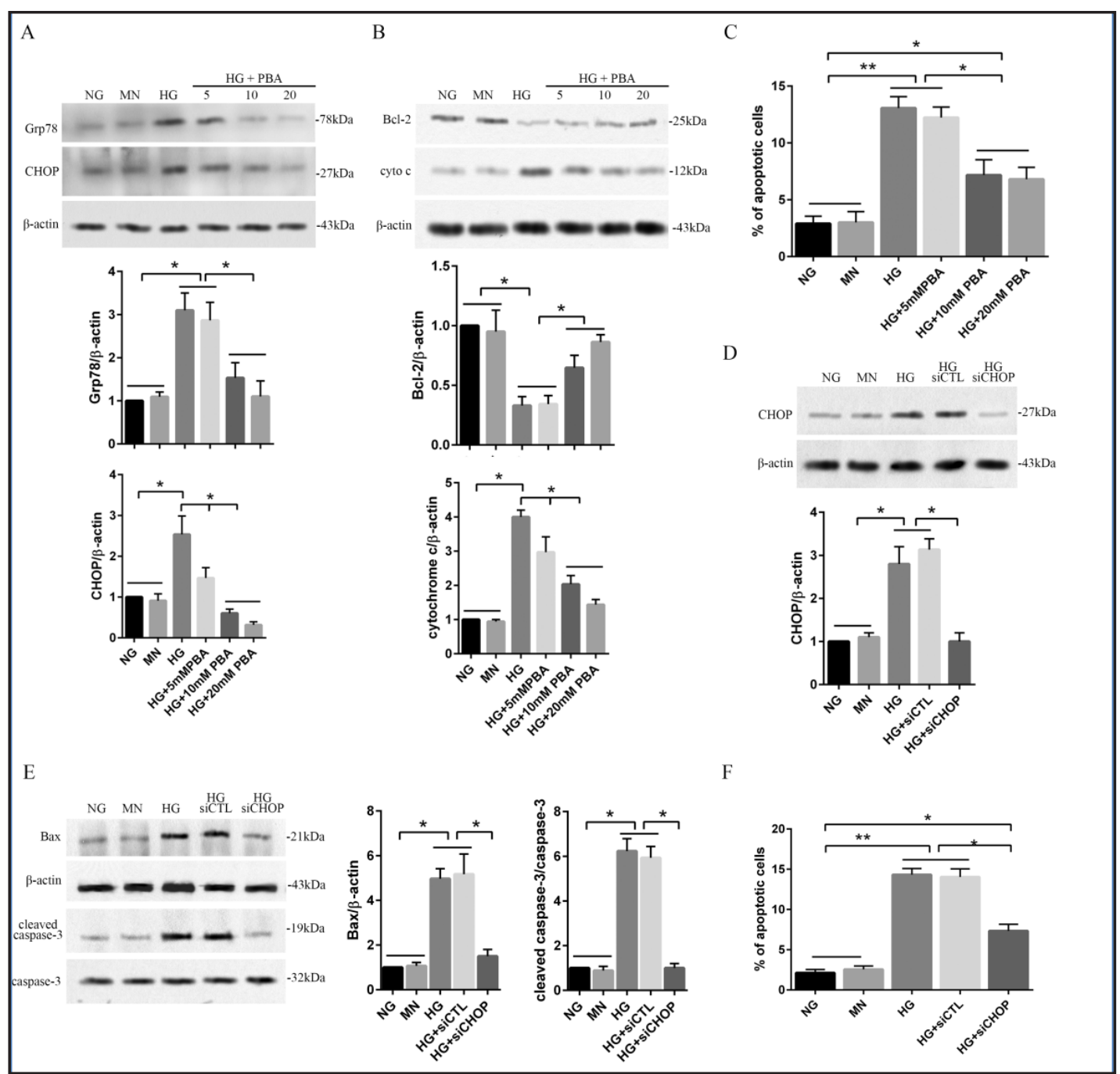

Fig. 4. Overwhelmed ER stress is related to HG-induced podocyte apoptosis. A - C. Podocytes were pretreated with the ER inhibitor sodium 4-phenylbultyrate (PBA: 5, 10, and $20 \mathrm{mM}$ ), and then $30 \mathrm{mM}$ high glucose (HG) was applied for $24 \mathrm{~h}$ in the presence or absence of PBA. Total cellular protein was extracted and immunoblot assay were performed for expression of CHOP and Grp78 (A) as well as Bcl-2 and cytochrome c (B). The effect of PBA on cellular apoptosis was assessed (C). D - F. Podocytes expressing CHOP siRNA (siCHOP) were treated with $30 \mathrm{mM} \mathrm{HG}$ for $24 \mathrm{~h}$. The siRNA that does not match any mouse gene sequences was used as knockdown control (siCTL). CHOP knockdown was confirmed using immunoblot assay (D). The abundance of Bax and the cleaved caspase-3 was assessed using immunoblot assay (E). Apoptosis was analyzed (F). Experiments were performed in triplicate. ${ }^{*}$ : $p<0.01$; $^{* *}$ : $<<0.001$.

mTOR activation is responsible for induction of ER stress and apoptosis in HG-treated podocytes

Apoptosis is also controlled and/or regulated by the other important cellular event, the mammalian target of rapamycin (mTOR) signaling [36]. It was reported that there is a crosstalk between ER stress and mTOR signaling in diverse cell types [19, 20]. Firstly, we detected increase of phosphorylated mTOR at Ser2448, not Ser2481 in HGtreated cells compared to NG and MN control (Fig. 5A). This indicates that mTOR complex 1 (mTORC1) is activated in HG- treated podocytes. The downstream signaling of mTORC1 is mediated by p70S6K, a mitogen activated Ser/Thr protein kinase required for cell 
Fig. 5. The mTOR signaling is over-activated in HG-treated podocytes. A, B. Podocytes were treated for $24 \mathrm{~h}$ with $30 \mathrm{mM}$ high glucose (HG). Normal glucose (NG: $5.6 \mathrm{mM}$ ) with or without mannitol (MN: $24.4 \mathrm{mM}$ ) was used as control. Total cellular protein was extracted and immunoblot assay was performed for evaluation of phospho-mTOR (A) and phospho-p70s6K (B), respectively. C. Podocytes were treated for the indicated time periods with $30 \mathrm{mM} \mathrm{HG}$ and 24.4 mM MN. Total cellular protein was extracted and immunoblot assay was performed for the evaluation of phosphoErk1/2 and total Erk1/2. D. Podocytes were pretreated with the Erk1/2 inhibitor U0126, and then 30 mM HG was applied for $24 \mathrm{~h}$ in the presence of $5 \mu \mathrm{M}$ U0126. Effects of U0126 on the phosphorylated mTOR at Ser2448 were assessed using immunoblot assay. Experiments were performed in triplicate. *: $\mathrm{p}<0.01$.
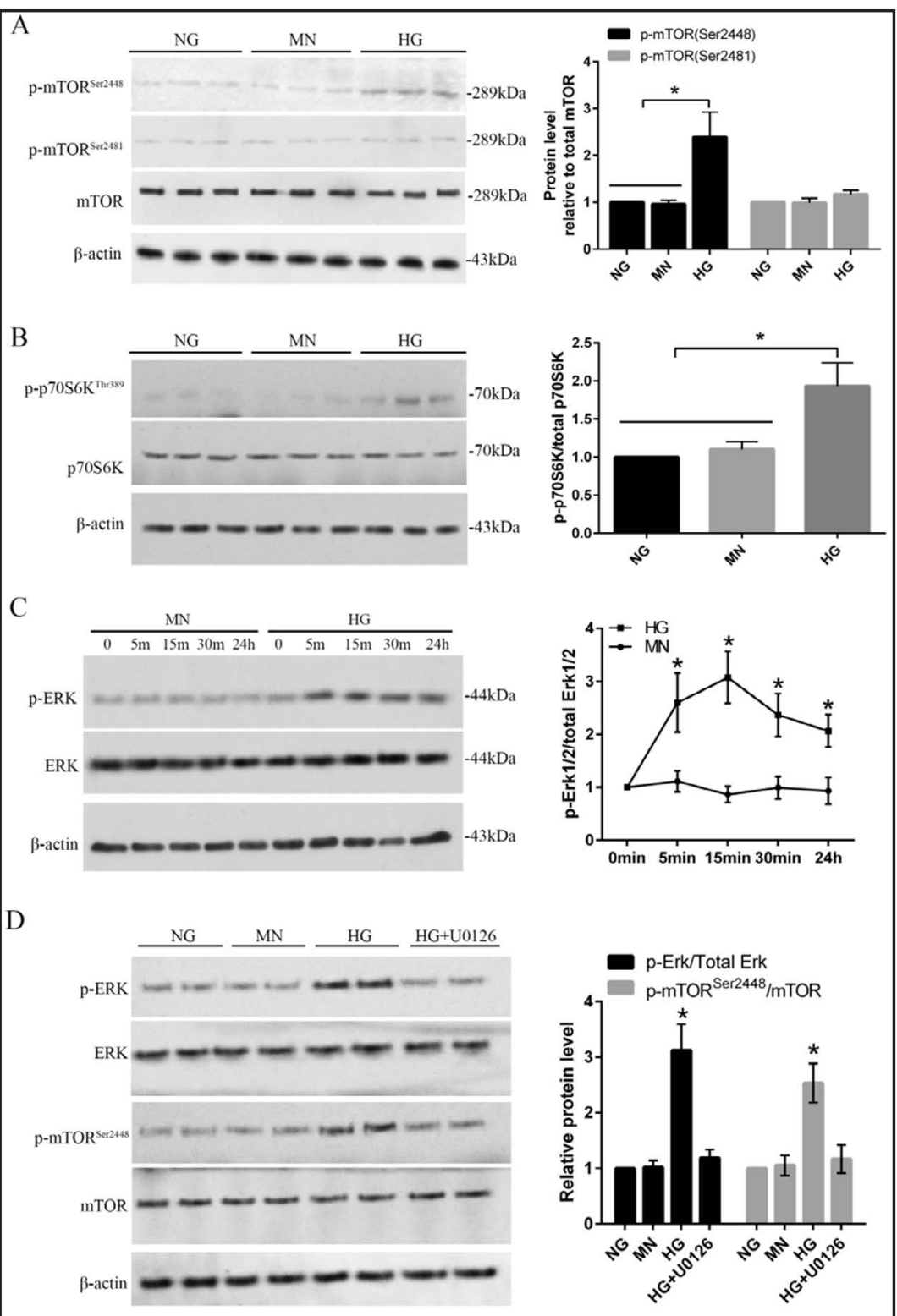

growth [36]. Phosphorylation of p70S6K at Thr389 is critical for its kinase function [36]. In this study, increased phospho-p70S6KThr389 was detected in HG-treated podocytes (Fig. 5B), indicating that signaling from the mTORC1 complex was enhanced following HG application. It has been reported that mTOR activated by Erk1/2 plays an important role in cancer progression [37]. We assessed the level of phospho-Erk1/2 in HG-treated podocytes. In comparison with osmotic mannitol control, rapid and persist activation of Erk1/2 was detected following HG treatment, as evidenced by phospho-Erk1/2 increased significantly at 5 min persisting to $24 \mathrm{~h}$ in HG-treated podocytes (Fig. 5C). Interestingly, the Erk1/2 inhibitor U0126 prevented increased activation of mTOR at Ser2448 in HG-treated podocytes (Fig. 5D). Thus, these results suggest that Erk1/2 is related to activation of mTOR under HG conditions in podocytes.

Finally, we inhibited mTOR signaling by the application of rapamycin, mainly targeting to mTORC1 complex [36]. In HG-treated cells, the phosphorylation level of mTORSer2448

\section{KARGER}


Fig. 6. mTOR activation is responsible for induction of ER stress and apoptosis in HG- treated podocytes. Podocytes were pretreated with mTOR inhibitor rapamycin, and then $30 \mathrm{mM}$ HG was applied for 24 $\mathrm{h}$ in the presence of $10 \mathrm{nM}$ rapamycin. A, B. Total cellular protein was extracted and immunoblot assay was performed for phospho-mTOR and phospho-p70S6K (A), CHOP and Grp78 (B). C. Podocytes were treated for the indicated time periods with $30 \mathrm{mM}$ high glucose (HG) and osmotic mannitol (MN) control. Total cellular protein was extracted and immunoblot assay was performed for evaluation of and phospho-AMPK $\alpha$ and total AMPK $\alpha$. D. Podocytes were pretreated with mTOR inhibitor rapamycin, and then $30 \mathrm{mM} \mathrm{HG}$ was applied for 24 $\mathrm{h}$ in the presence of $10 \mathrm{nM}$ rapamycin. Effects of rapamycin on phosphoAMPK $\alpha$ level were assessed using immunoblot assay. E. The effect of rapamycin on cellular apoptosis was assessed with annexin V-FITC and propidium iodide staining. Experiments were performed in triplicate. *: $\mathrm{p}<0.01,{ }^{\#}$ : $\mathrm{P}<0.05$.

Lei et al.: mTOR Activation and ER Stress in HG Podocyte Injury

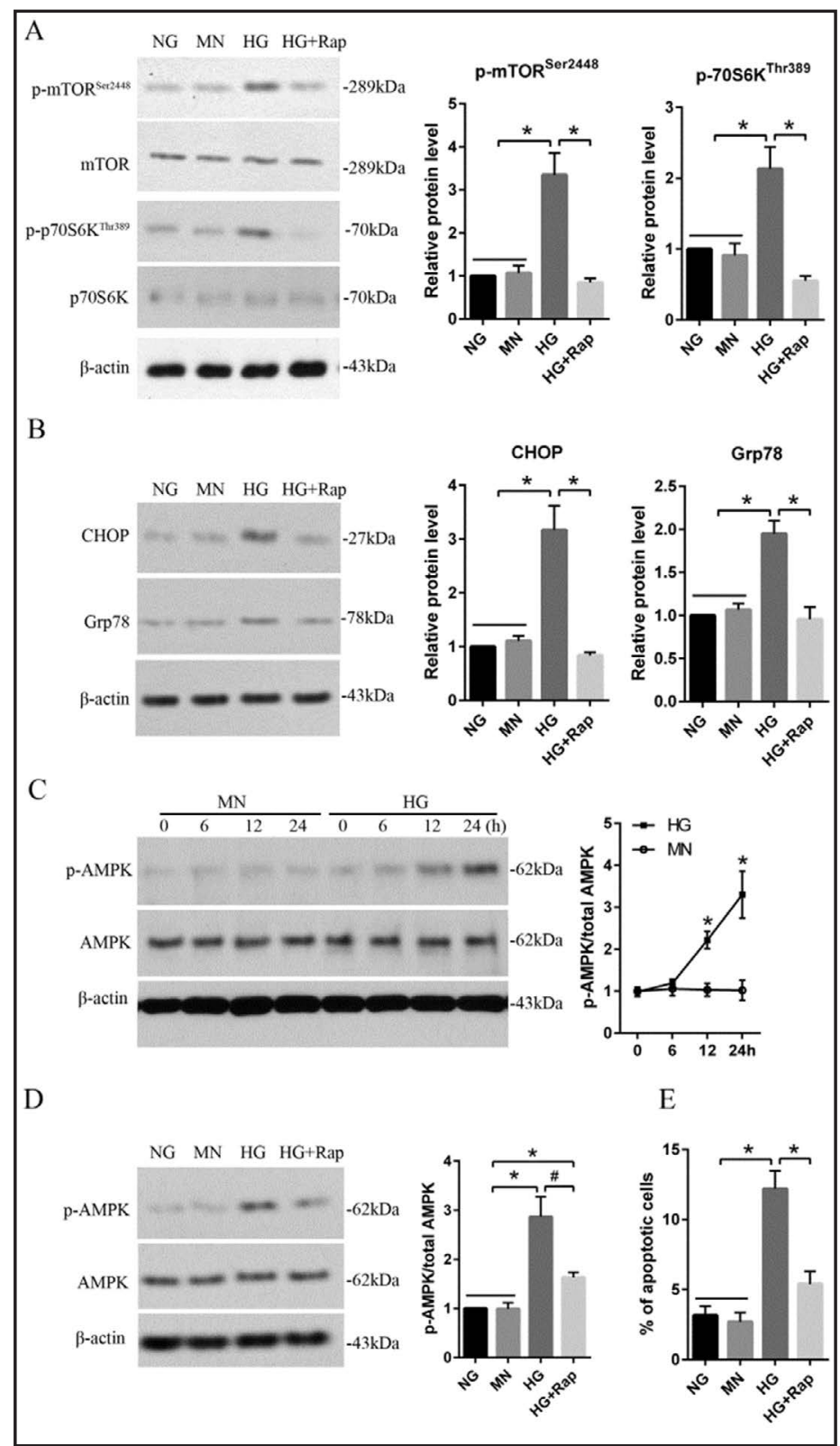

and p70S6KThr389 did not increase in the presence of rapamycin (Fig. 6A). Moreover, induction of CHOP and Grp78 was inhibited by rapamycin in HG-treated cells (Fig. 6B). In rat puromycin nephrosis model, it has been reported that mTORC1 was activated and thus led to energy consumption which in turn activated ER stress. The overwhelmed ER stress was responsible for podocyte damage and proteinuria development [38]. In the current study, the activation level of AMPK $\alpha$ that is regarded as the sensor of the AMP/ATP ratio within cells was evaluated. Under HG conditions, phospho-AMPK $\alpha$ was increased significantly at 12 and $24 \mathrm{~h}$ (Fig. 6C). Increased phospho-AMPK $\alpha$ was prevented remarkably by rapamycin in HG-treated podocytes (Fig. 6D). Notably, flow cytometry showed that the application of rapamycin also significantly (HG+Rap $5.4 \pm 0.90 v s$ HG $12.20 \pm 1.28, p<0.001$ ) decreased HG-induced apoptosis (Fig. 6E). These findings suggest that enhanced mTOR signaling contributes to ER stress activation by increasing energy consumption, which is responsible for induction of apoptosis in HG-treated podocytes. 


\section{Cellular Physiology Cell Physiol Biochem 2018;45:2431-2443 \\ and Biochemistry Published online: March 19, $2018 \quad \begin{aligned} & \text { DOI: 10.1159/000488231 } 2018 \text { The Author(s). Published by S. Karger AG, Basel } \\ & \text { www.karger.com/cpb }\end{aligned}$}

Lei et al.: mTOR Activation and ER Stress in HG Podocyte Injury

\section{Discussion}

Podocyte loss plays a critical role in the pathogenesis of proteinuria in DN [1, 2, 5-7]. As reported previously [8-11, 24, 25], mouse podocytes are damaged under HG culture conditions. We provide evidence that ER stress and mTOR signaling is involved in HGinduced podocyte injury.

ER stress is also named as unfolded protein response (UPR) since it mainly deal with unfolded and/or miss-folded proteins $[16,17]$. In the conditions of over-activated and/or prolongedERstress, the role of UPR changes from promoting cellular survival to committing the cell to an apoptotic pathway. The C/EBP homologous protein (CHOP), also known as GADD153, is a transcription factor that is activated at multiple levels during ER stress [16, 17]. Here, we found that the protein levels of CHOP and Grp78/BiP, the other representative marker of ER stress [31], were increased in HG-treated podocytes. In both Heymann nephritis, a membranous nephropathy model, and puromycin nephrosis, a minimal change nephrotic model, the induction of ER stress in podocytes have been reported, showing increased expression of ER chaperones Grp78 and Grp94 [39, 40]. Upon severe ER stress, Grp78 is released from three major ER resident transducers also named as UPR receptors, PERK, IRE-1, and activating transcription factor 6 (ATF6) [32]. PERK dimerizes with Grp78 in resting cells, and oligomerizes and thus is activated in ER-stressed cells. Activated PERK phosphorylates the alpha subunit of eukaryotic translation- initiation factor 2 (EIF2) and causes its inactivation and thus a rapid reduction of translational initiation and repression of global protein synthesis, leading to growth arrest [41]. In HG-treated podocytes, the phospho-PERKThr982 was remarkably upregulated. Downstream pathways of all the three UPR receptors have been identified as having pro-apoptotic roles, leading to cell death [7, 35]. It has been shown that caspase- 12 appears to be necessary for apoptosis induced by a variety of ER-directed pro-apoptosis signals [28-30]. In addition, CHOP can cause downregulation of the anti-apoptotic mitochondrial protein Bcl-2, favoring a pro-apoptotic drive at the mitochondria by inducing cytochrome c release and caspase- 3 activation [31, 32]. In comparison with $\mathrm{NG}$ and $\mathrm{MN}$ controls, the level of Bcl-2 is decreased while cytochrome $\mathrm{c}$ is increased in HG-treated podocytes. Nevertheless, other membranes of Bcl-2 family exhibit pro- apoptotic activities, such as Bax and Bak that are extensively expressed in different tissues [33-35]. Recruitment of Bax and Bak to mitochondria outer membrane initiates the release of cytochrome c from mitochondria to cytosol, leading to induction of apoptotic pathway [33-35]. In our study, upregulation of Bax and Bak was detected in HGtreated podocytes. These findings suggest that enhanced ER stress and over-activation of its downstream effector may be related to HG-induced podocyte apoptosis.

To further clarify the role of ER stress in HG-induced podocyte injury, we blocked ER stress by using either a specific inhibitor sodium 4-phenylbultyrate (PBA) or siCHOP for knockdown of CHOP expression. As expected, inhibition of ER stress remarkably decreased HG-induced podocyte apoptosis, indicating that over-activated ER stress plays a crucial role in induction of podocyte apoptosis under HG conditions. Nevertheless, it should be noted that the ability of CHOP in inducing apoptosis is dependent on the duration and degree of ER stress. In vivo evidence from puromycin nephrosis model demonstrates that podocyte damage was associated with ER stress, which in turn caused biogenesis reduction of nephrin, a key podocyte slit diaphragm protein [40]. Therefore, dysfunctional ER stress in podocytes not only contributes to podocyte apoptosis, but also to podocyte structural protein synthesis possibly through its downstream transcription factors such as ATF6.The mTOR signaling, another important cellular event, is shown to have pro-apoptotic role by interacting with ER stress response [42]. The pro- apoptotic role of mTOR is coupled to the downstream activation of both PERK and IRE-1 under ER stress conditions [43-45]. It was found that in podocytes, the mTOR complex 1 (mTORC1) activation plays a critical role in the development of mice DN [46, 47]. In HG-treated podocytes, the level of phosphomTORSer2448 representing mTORC1 activation and its downstream effector phosphop70S6kThr389 was increased significantly. This indicates that the mTOR signaling is 


\section{Cellular Physiology Cell Physiol Biochem 2018;45:2431-2443

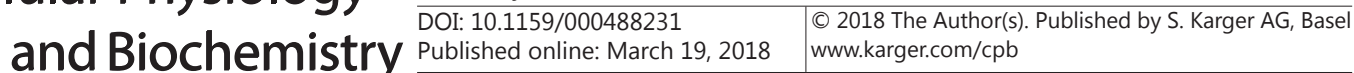 \\ Lei et al.: mTOR Activation and ER Stress in HG Podocyte Injury}

activated in HG-induced podocyte injury. It was reported that the down-regulation of mTOR activity by rapamycin enhances cell viability under ER stress conditions $[43,48,49]$. In the current study, the administration of rapamycin significantly prevented the upregulation of CHOP and Grp78. In consistent, it was reported that rapamycin decreased the levels of CHOP/GADD153, one of the representatives of ER stress, in tunicamycin-treated podocyte [50]. We also found that HG-induced apoptosis was decreased by rapamycin. These findings provide evidence that HG condition resulted in mTOR activation, which may be related to over-activated ER stress and podocyte apoptosis. In puromycin nephrosis model, it was found that mTORC1 was activated, which in turn activated ER stress by energy consumption and induced podocyte damage and proteinuria [38]. In HG-treated podocytes, we also analyzed the level of energy consumption by assessing the abundance of phosphorylated AMPK $\alpha$, a sensor of AMP/ATP ratio within cells. Increased phosphoAMPK $\alpha$ was detected in HG podocytes, which was prevented by rapamycin. This implies that following HG treatment over-activated mTOR signaling may induce ER stress through enhancing energy consumption. Although increased mTOR activation can cause ER stress potentially by increasing unfolded/misfolded protein load in the ER and thus consuming more energy, the activated UPR or ER stresses can subsequently downregulate mTOR activation as a negative feedback [51]. Actually, the interconnection between ER stress and mTOR is complicated since the UPR activation can occur downstream or upstream of the mTOR signaling. Contradictory findings were reported that mTOR inhibition by rapamycin aggravated proteinuria in puromycin nephrosis, and led to decrease of the slit diaphragm proteins expression in cultured podocytes $[52,53]$. Therefore, under HG conditions, the role of mTOR and the relationship with ER stress needs be deeply investigated by designing delicate experiments, particularly with different start point for rapamycin treatment.

The mechanism by which mTORC1 is activated in HG-treated podocytes was explored preliminarily. Previous study reported that TGF- $\beta 1$ induced podocyte apoptosis through Erk- mediated activation of mTORC1/Nox4 axis [54]. The other study found that under HG conditions in podocytes Erk was activated which is responsible for increased VEGF expression [55]. These findings suggest that Erk pathway may be related to HG-induced mTOR activation in podocytes. Following HG treatment, the phospho-Erk level was rapidly increased with persistent to $24 \mathrm{~h}$. Inhibition of Erk by U0126 prevented HG-induced mTOR activation in cultured podocytes. Therefore, Erk pathway is related to mTOR activation under HG conditions in podocytes. Nevertheless, additional studies should be performed to further disclose the mechanism of mTOR activation since other signal pathway such as JNK is also associated with mTIR activation.

\section{Conclusion}

Taken together, we demonstrate that overwhelmed ER stress signaling by Erk-mediated mTOR activation is involved in HG-induced podocyte apoptosis. Nevertheless, it should be further investigated whether the findings that we obtained from HG-treated podocytes can be applied to in vivo DN and non-diabetic conditions.

\section{Acknowledgements}

This work was supported by the Central Laboratory of the First Hospital of Jilin University.

\section{Disclosure Statement}

The authors have nothing to disclose. 


\section{Cellular Physiology Cell Physiol Biochem 2018;45:2431-2443

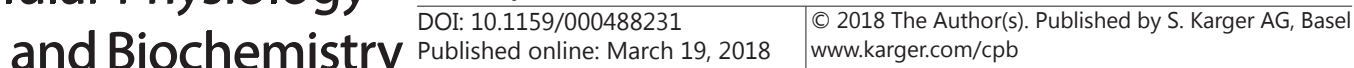

Lei et al.: mTOR Activation and ER Stress in HG Podocyte Injury

\section{References}

1 Lin JS, Susztak K: Podocytes: the Weakest Link in Diabetic Kidney Disease? Curr Diab Rep 2016;16:45.

-2 Reidy K, Kang HM, Hostetter T, Susztak K: Molecular mechanisms of diabetic kidney disease. J Clin Invest 2014;124:2333-2340.

- 3 Perico L, Conti S, Benigni A, Remuzzi G: Podocyte-actin dynamics in health and disease. Nat Rev Nephrol. 2016;12:692-710.

4 Kawanami D, Matoba K, Utsunomiya K: Signaling pathways in diabetic nephropathy. Histol Histopathol 2016;31:1059-1067.

5 Liu WT, Peng FF, Li HY, Chen XW, Gong WQ Chen WJ, Chen YH, Li PL, Li ST, Xu ZZ, Long HB: Metadherin facilitates podocyte apoptosis in diabetic nephropathy. Cell Death Dis 2016;7:e2477.

6 Eid S, Boutary S, Braych K, Sabra R, Massaad C, Hamdy A, Rashid A, Moodad S, Block K, Gorin Y, Abboud HE, Eid AA: mTORC2 Signaling Regulates Nox4-Induced Podocyte Depletion in Diabetes. Antioxid Redox Signal 2016;25:703-719.

7 Cao AL, Wang L, Chen X, Wang YM, Guo HJ, Chu S, Liu C, Zhang XM, Peng W: Ursodeoxycholic acid and 4-phenylbutyrate prevent endoplasmic reticulum stress-induced podocyte apoptosis in diabetic nephropathy. Lab Invest 2016;96:610-622.

8 Zhang Y, Gao X, Chen S, Zhao M, Chen J, Liu R, Cheng S, Qi M, Wang S, Liu W: Cyclin- dependent kinase 5 contributes to endoplasmic reticulum stress induced podocyte apoptosis via promoting MEKK1 phosphorylation at Ser280 in diabetic nephropathy. Cell Signal 2017;31:31-40.

-9 Shi JX, Wang QJ, Li H, Huang Q: SIRT4 overexpression protects against diabetic nephropathy by inhibiting podocyte apoptosis. Exp Ther Med 2017;13:342-348.

10 Langer S, Kreutz R, Eisenreich A: Metformin modulates apoptosis and cell signaling of human podocytes under high glucose conditions. J Nephrol 2016;29:765-773.

11 Susztak K, Raff AC, Schiffer M, Böttinger EP: Glucose-induced reactive oxygen species cause apoptosis of podocytes and podocyte depletion at the onset of diabetic nephropathy. Diabetes 2006;55:225-233

12 Schiffer M, Bitzer M, Roberts IS, Kopp JB, ten Dijke P, Mundel P, Böttinger EP: Apoptosis in podocytes induced by TGF-beta and Smad7. J Clin Invest 2001;108:807-816.

13 Li JH, Huang XR, Zhu HJ, Oldfield M, Cooper M, Truong LD, Johnson RJ, Lan HY: Advanced glycation end products activate Smad signaling via TGF-beta-dependent and independent mechanisms: implications for diabetic renal and vascular disease. FASEB J 2004;18:176-178.

14 Hartleben B, Gödel M, Meyer-Schwesinger C, Liu S, Ulrich T, Köbler S, Wiech T, Grahammer F, Arnold SJ, Lindenmeyer MT, Cohen CD, Pavenstädt H, Kerjaschki D, Mizushima N, Shaw AS, Walz G, Huber TB: Autophagy influences glomerular disease susceptibility and maintains podocyte homeostasis in aging mice. J Clin Invest 2010;120:1084-1096

-15 Miranda-Díaz AG, Pazarín-Villaseñor L, Yanowsky-Escatell FG, Andrade-Sierra J: Oxidative Stress in Diabetic Nephropathy with Early Chronic Kidney Disease. J Diabetes Res 2016;2016:7047238.

16 Lee WS, Yoo WH, Chae HJ: ER Stress and Autophagy. Curr Mol Med 2015;15:735-745.

17 Song S, Tan J, Miao Y, Li M, Zhang Q: Crosstalk of Autophagy and Apoptosis: Involvement of the Dual Role of Autophagy Under ER Stress. J Cell Physiol 2017; 232:2977-2984.

-18 Wang B, Xu X, He X, Wang Z, Yang M: Berberine improved Aldo-induced podocyte injury via inhibiting oxidative stress and endoplasmic reticulum stress pathways both in vivo and in vitro. Cell Physiol Biochem 2016;39:217-228.

19 Heberle AM, Prentzell MT, van Eunen K, Bakker BM, Grellscheid SN, Thedieck K: Molecular mechanisms of mTOR regulation by stress. Mol Cell Oncol 2014;2:e970489.

20 Dong G, Liu Y, Zhang L, Huang S, Ding HF, Dong Z: mTOR contributes to ER stress and associated apoptosis in renal tubular cells. Am J Physiol Renal Physiol 2015;308:F267-F274.

21 Zhang HT, Wang WW, Ren LH, Zhao XX, Wang ZH, Zhuang DL, Bai YN: The mTORC2/Akt/NFkB pathwaymediated activation of TRPC6 participates in adriamycin- induced podocyte apoptosis. Cell Physiol Biochem 2016;40:1079-1093.

22 Liu Y, Su L, Lin Q, Han Y, You P, Fan Q: Induction of c-mip by IL-17 plays an important role in adriamycininduced podocyte damage. Cell Physiol Biochem 2015;36:1274-1290. 


\section{Cellular Physiology Cell Physiol Biochem 2018;45:2431-2443

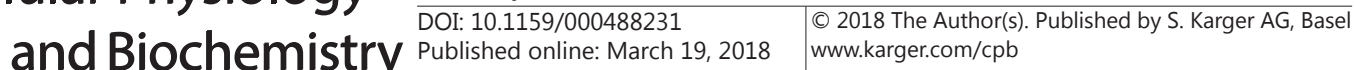

-23 Yu L, Liu Y, Wu Y, Liu Q, Feng J, Gu X, Xiong Y, Fan Q, Ye J: Smad3/Nox4-mediated mitochondrial dysfunction plays a crucial role in puromycin aminonucleoside-induced podocyte damage. Cell Signal 2014;26:2979-2991.

-24 Jin Y, Liu S, Ma Q Xiao D, Chen L: Berberine enhances the AMPK activation and autophagy and mitigates high glucose-induced apoptosis of mouse podocytes. Eur J Pharmacol 2017;794:106-114.

-25 Jo HA, Kim JY, Yang SH, Han SS, Joo KW, Kim YS, Kim DK: The role of local IL6/JAK2/STAT3 signaling in high glucose-induced podocyte hypertrophy. Kidney Res Clin Pract 2016;35:212-218.

-26 Brosius FC, Coward RJ: Podocytes, signaling pathways, and vascular factors in diabetic kidney disease. Adv Chronic Kidney Dis 2014;21:304-310.

27 Savitskaya MA, Onishchenko GE: Mechanisms of Apoptosis. Biochemistry (Mosc) 2015;80:1393-1405.

28 Shiraishi H, Okamoto H, Yoshimura A, Yoshida H: ER stress-induced apoptosis and caspase-12 activation occurs downstream of mitochondrial apoptosis involving Apaf-1_ J Cell Sci 2006;119:3958-3966.

-29 Morishima N, Nakanishi K, Takenouchi H, Shibata T, Yasuhiko Y: An endoplasmic reticulum stress-specific caspase cascade in apoptosis. Cytochrome c-independent activation of caspase- 9 by caspase-12. J Biol Chem 2002;277:34287-34294.

-30 Szegezdi E, Fitzgerald U, Samali A: Caspase-12 and ER-stress-mediated apoptosis: the story so far. Ann N Y Acad Sci 2003;1010:186-194.

-31 Li Y, Guo Y, Tang J, Jiang J, Chen Z: New insights into the roles of CHOP-induced apoptosis in ER stress. Acta Biochim Biophys Sin (Shanghai) 2014;46:629-640.

-32 Rodriguez D, Rojas-Rivera D, Hetz C: Integrating stress signals at the endoplasmic reticulum: The BCL-2 protein family rheostat. Biochim Biophys Acta 2011;1813:564-574.

-33 Szegezdi E, Logue SE, Gorman AM, Samali A: Mediators of endoplasmic reticulum stress-induced apoptosis. EMBO Rep 2006; 7:880-885.

34 Tabas I, Ron D: Integrating the mechanisms of apoptosis induced by endoplasmic reticulum stress. Nat Cell Biol 2011;13:184-190.

-35 Sano R, Reed JC: ER stress-induced cell death mechanisms. Biochim Biophys Acta 2013;1833:3460-3470.

-36 Laplante M, Sabatini DM: mTOR signaling at a glance. J Cell Sci 2009;122:3589-3594.

-37 Linda S. Steelman,William H. Chappell, Stephen L. Abrams, C. Ruth Kempf,Jacquelyn Long,Piotr Laidler, Sanja Mijatovic, Danijela Maksimovic-Ivanic, Franca Stivala, Maria C. Mazzarino, Marco Donia, Paolo Fagone, Graziella Malaponte, Ferdinando Nicoletti, Massimo Libra, Michele Milella, Agostino Tafuri, Antonio Bonati, Jörg Bäsecke, Lucio Cocco, Camilla Evangelisti, Alberto M. Martelli, Giuseppe Montalto, Melchiorre Cervello, James A. McCubrey: Roles of the Raf/MEK/ERK and PI3K/PTEN/Akt/mTOR pathways in controlling growth and sensitivity to therapy-implications for cancer and aging. Aging (Albany NY) 2011; 3: 192-222.

-38 Ito N, Nishibori Y, Ito Y, Takagi H, Akimoto Y, Kudo A, Asanuma K, Sai Y, Miyamoto K, Takenaka H, Yan K: mTORC1 activation triggers the unfolded protein response in podocytes and leads to nephrotic syndrome. Lab Invest 2011;91:1584-1595. Cybulsky AV, Takano T, Papillon J, Bijian K: Role of the endoplasmic reticulum unfolded protein response in glomerular epithelial cell injury. J Biol Chem 2005;280:2439624403.

39 Cybulsky AV, Takano T, Papillon J, Bijian K: Role of the endoplasmic reticulum unfolded protein response in glomerular epithelial cell injury. J Biol Chem 2005;280:24396-24403.

40 Nakajo A, Khoshnoodi J, Takenaka H, Hagiwara E, Watanabe T, Kawakami H, Kurayama R, Sekine Y, Bessho F, Takahashi S, Swiatecka-Urban A, Tryggvason K, Yan K: Mizoribine corrects defective nephrin biogenesis by restoring intracellular energy balance. J Am Soc Nephrol 2007;18:2554-2564.

41 Liu Z, Cai H, Zhu H, Toque H, Zhao N, Qiu C, Guan G, Dang Y, Wang J: Protein kinase RNA-like endoplasmic reticulum kinase (PERK)/calcineurin signaling is a novel pathway regulating intracellular calcium accumulation which might be involved in ventricular arrhythmias in diabetic cardiomyopathy. Cell Signal 2014;26:2591-2600.

-42 Appenzeller-Herzog C, Hall MN: Bidirectional crosstalk between endoplasmic reticulum stress and mTOR signaling. Trends Cell Biol 2012;22:274-282.

-43 Kato H, Nakajima S, Saito Y, Takahashi S, Katoh R: mTORC1 serves ER stress-triggered apoptosis via selective activation of the IRE1-JNK pathway. Cell Death Differ 2012;19:310-320. 


\section{Cellular Physiology Cell Physiol Biochem 2018;45:2431-2443

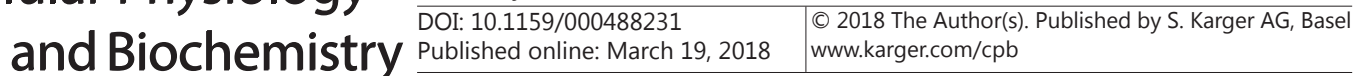 \\ Lei et al.: mTOR Activation and ER Stress in HG Podocyte Injury}

44 Ozean U, Ozcan L, Yilmaz E, Duvel K, Sahin M: Loss of the tuberous sclerosis complex tumor suppressors triggers the unfolded protein response to regulate insulin signaling and apoptosis. Mol Cell 2008;29:541-551.

45 Hyrskyluoto A, Reijonen S, Kivinen J, Lindholm D, Korhonen L: GADD34 mediates cytoprotective autophagy in mutant huntingtin expressing cells via the mTOR pathway. Exp Cell Res 2012;318:33-42.

-46 Gödel M, Hartleben B, Herbach N, Liu S, Zschiedrich S, Lu S, Debreczeni-Mór A, Lindenmeyer MT, Rastaldi MP, Hartleben G, Wiech T, Fornoni A, Nelson RG, Kretzler M, Wanke R, Pavenstädt H, Kerjaschki D, Cohen CD, Hall MN, Rüegg MA, Inoki K, Walz G, Huber TB: Role of mTOR in podocyte function and diabetic nephropathy in humans and mice. J Clin Invest 2011;121:2197-2209.

47 Inoki K, Mori H, Wang J, Suzuki T, Hong S, Yoshida S, Blattner SM, Ikenoue T, Rüegg MA, Hall MN, Kwiatkowski DJ, Rastaldi MP, Huber TB, Kretzler M, Holzman LB, Wiggins RC, Guan KL: mTORC1 activation in podocytes is a critical step in the development of diabetic nephropathy in mice. J Clin Invest 2011;121:2181-2196.

48 Ravikumar B, Berger Z, Vacher C, O’Kane CJ, Rubinsztein DC; Rapamycin pre-treatment protects against apoptosis. Hum Mol Genet 2006;15:1209-1216.

49 Ogata M, Hino S, Saito A, Morikawa K, Kondo S: Autophagy is activated for cell survival after endoplasmic reticulum stress. Mol Cell Biol 2006;26:9220-9231.

50 Cheng YC, Chang JM, Chen CA, Chen HC: Autophagy modulates endoplasmic reticulum stress-induced cell death in podocytes: a protective role. Exp Biol Med 2015;240:467-476.

51 Wouters BG, Koritzinsky M: Hypoxia signaling through mTOR and the unfolded protein response in cancer. Nat Rev Cancer 2008;8:851-864.

-52 Vollenbroker B, George B, Wolfgart M, Saleem MA, Pavenstädt H, Weide T: mTOR regulates expression of slit diaphragm proteins and cytoskeleton structure in podocytes. Am J Physiol Renal Physiol 2009;296:F418-F422.

53 Torras J, Herrero-Fresneda I, Gulias O, Flaquer M, Vidal A, Cruzado JM, Lloberas N, Franquesa Ml, Grinyó JM: Rapamycin has dual opposing effects on proteinuric experimental nephropathies: is it a matter of podocyte damage? Nephrol Dial Transplant 2009;24:3632-3640.

54 Das R, Xu S, Nguyen TT, Quan X, Choi SK, Kim SJ, Lee EY, Cha SK, Park KS: Transforming growth factor $\beta 1$-induced apoptosis in podocytes via the extracellular signal-regulated kinase-mammalian target of rapamycin complex 1-NADPH Oxidase 4 Axis. J Biol Chem 2015;290:30830-30842.

55 Hoshi S, Nomoto K, Kuromitsu J, Tomari S, Nagata M: High glucose induced VEGF expression via PKC and ERK in glomerular podocytes. Biochem Biophys Res Commun 2002;290:177-184. 